\title{
Diretrizes para terapia nutricional em crianças com câncer em situação crítica
}

\author{
Guidance of nutritional support in critically ill \\ children with cancer
}

Adriana GARÓFOLO'

\section{R E S U M O}

Este trabalho é constituído de uma revisão de conceitos fundamentais e atualizados relacionados à terapia nutricional de crianças com câncer criticamente doentes. O objetivo principal desta revisão é discutir alterações nutricionais e metabólicas decorrentes dessa condição, bem como as indicações, recomendações, contra-indicações e complicações da terapia nutricional (nutrição enteral e parenteral) em pacientes com câncer. Este artigo aborda, de forma objetiva, a aplicação prática de alguns conceitos baseados em evidências científicas, e propõe algumas diretrizes para auxiliar na decisão da terapia nutricional no âmbito da vivência clínica. Conclui-se que há necessidade de maior incentivo ao desenvolvimento da ciência da terapia de suporte, como o tratamento das infecções, a terapia intensiva e a terapia metabólico-nutricional, para que se ampliem as possibilidades de cura de crianças e adolescentes com câncer.

Termos de indexação: criança, neoplasias, nutrição enteral, nutrição parenteral, terapia nutricional.

\section{A B S T R A C T}

This paper is a review of fundamental, up to date concepts of nutritional support for critically ill children with cancer. The aim of the text is to discuss the nutritional and metabolic changes caused by their condition, as well as indications and recommendations, contraindications and complications of nutritional support (enteral and parenteral nutrition) in cancer patients. The paper objectively shows the practical application of scientific knowledge and gives guidance to improve decisions about nutritional support in clinical practice. Finally, it is concluded there is a need to encourage research on support therapy, such as for the treatment of infections and for intensive nutritional and metabolic support, so as to increase the chances of curing children and adolescents with cancer.

Indexing terms: child, neoplasms, enteral nutrition, parenteral nutrition, nutritional therapy.

\footnotetext{
1 Departamento de Pediatria, Serviço de Nutrição Clínica, Grupo de Apoio ao Adolescente e à Criança com Câncer, Instituto de Oncologia Pediátrica, Universidade Federal de São Paulo. Rua Botucatu, 743, Vila Clementino, 04023-062, São Paulo, SP, Brasil. E-mail: <adrigarofolo@hotmail.com>.
} 


\section{N T R O D U Ç Ã O}

O câncer infantil compreende 0,5\% a 3,0\% de todas as neoplasias malignas humanas na maioria das populações ${ }^{1}$, estimando-se uma incidência anual de cerca de duzentos mil casos em todo o mundo. Nessa ordem, as principais regiões do globo são Uganda na África, Croácia, Nova Zelândia, Dinamarca, Estados Unidos e Canadá, sendo, normalmente, superior no sexo masculino ${ }^{2}$. Entre os tumores mais comuns da infância estão as leucemias, com incidência de aproximadamente $30,0 \%$ dos casos, seguidas pelos tumores do sistema nervoso central (19,0\%), linfomas $(13,0 \%)$, neuroblastoma $(8,0 \%)$, sarcomas de partes moles (7,0\%), tumor de Wilms $(6,0 \%)$, tumores ósseos $(5,0 \%)$ e retinoblastoma $(3,0 \%)^{2}$. Em Goiânia, Brasil, destacam-se os tumores ósseos em meninas, com taxas de incidência anual entre as maiores do mundo $(11,7 / \text { milhão de habitantes menores de } 15 \text { anos })^{3}$.

As formas mais comuns de tratamento antineoplásico incluem quimioterapia, radioterapia, cirurgia e transplante de medula óssea. O tratamento, assim como a própria doença, têm efeitos agressivos para o hospedeiro, deixando o organismo vulnerável e debilitado, aumentando o risco de comprometimento nutricional. A desnutrição, quando presente, agrava ainda mais o quadro clínico, prejudicando a resposta terapêutica ${ }^{4-6}$.

A prevalência de desnutrição ao diagnóstico de crianças com câncer tem demonstrado uma variação de $6 \%$ a $50 \%$, considerando diferentes estudos. Essa variabilidade é conseqüência da heterogeneidade do grupo - diagnóstico e estágio da doença - bem como da heterogeneidade dos critérios utilizados para classificar a desnutrição. Em geral, o maior risco nutricional está associado aos tumores sólidos, principalmente nas doenças avançadas com algum grau de desnutrição presente logo no início do tratamento 7 .

Considerando todas as formas de desnutrição, o acompanhamento de 145 crianças e adolescentes durante a fase de indução da remissão do tratamento oncológico demonstrou 38\% de desnutrição energético-protéica (DEP) no estudo realizado num centro de referência em São Paulo8. De forma geral, o maior risco para desnutrição durante o tratamento está associado à terapia com múltiplas drogas quimioterápicas em altas doses e sua combinação com radioterapia, principalmente abdominal ou pélvica.

Determinados diagnósticos tendem a apresentar aumento nas taxas de DEP, bem como na sua gravidade, durante a terapia antineoplásica. Num estudo que acompanhou vinte adolescentes portadores de osteossarcomas, observou-se aumento de $10 \%$ na prevalência de DEP pelo índice de massa corpórea e pelas pregas cutâneas do tríceps, e 15\% pela circunferência muscular do braço, comparando o primeiro com o terceiro mês de tratamento. Além disso, houve agravo do estado nutricional, com redução significativa na média de adequação sobre o peso ideal, apesar da intervenção dietética. Provavelmente, esse efeito tenha ocorrido como conseqüência do uso de drogas quimioterápicas que prejudicam a ingestão alimentar e promovem perdas nutricionais importantes por toxicidade renal e gastrintestinal, principalmente vômitos intensos ${ }^{9}$.

Levando-se em consideração todas essas condições, é fácil imaginar que crianças com câncer tenham importantes alterações da condição nutricional. Por esse motivo, durante muitos anos, a nutrição parenteral (NP) foi a forma de terapia nutricional mais utilizada, especialmente entre os que recebem transplante de medula óssea (TMO) ou realizam ciclos intensivos de quimioterapia. Essa conduta decorre da ingestão oral prejudicada associada, principalmente, às toxicidades dos medicamentos sobre o trato gastrointestinal ${ }^{10-13}$. Atualmente, entretanto, tal conduta está sendo substituída pelo uso de sondas nasoenterais, particularmente nas nações mais desenvolvidas do globo, por ser factível, segura e bem tolerada ${ }^{12,14}$. 
Embora a nutrição enteral em crianças com câncer e após TMO apresente muitas vantagens, incluindo menor risco e custo do que a NP, efetividade na prevenção da depleção nutricional, redução dos episódios febris e de hemoculturas positivas ${ }^{15}$, ela tem recebido pouca importância dos profissionais dessa área no Brasil.

Apesar das vantagens, alguns aspectos ainda não foram totalmente elucidados, como a influência da nutrição enteral na resposta terapêutica e no prognóstico de cura das crianças com câncer, especialmente em TMO e em unidades de cuidados intensivos $(\mathrm{UCl})^{16}$. Por outro lado, diretrizes para auxiliar na decisão da terapia nutricional têm sido estabelecidas, bem como seus riscos. Esses aspectos serão abordados neste trabalho.

\section{A SPECTOS META B Ó LICOS}

\section{Resposta metabólica à agressão}

Pacientes gravemente doentes desenvolvem uma resposta aguda a uma variedade de estímulos locais e sistêmicos, que é traduzida por alterações endócrino-metabólicas e imunológicas, caracterizando o estado de estresse ${ }^{17}$.

Na resposta inflamatória à agressão, principalmente na sepse, duas fases podem ser identificadas, as quais conduzem à ineficiência nos mecanismos de defesa do hospedeiro: fase hiperinflamatória, caracterizada pela síndrome da resposta inflamatória sistêmica (SIRS), e fase hipoinflamatória, caracterizada pela síndrome da anti-resposta compensatória (CARS). Na SIRS, a resposta hiperinflamatória induz à hiperatividade dos leucócitos, com dano orgânico secundário. Já na CARS, há uma resposta hipoinflamatória que leva à redução da capacidade dos leucócitos em destruir microrganismos, numa tentativa de conter a resposta pró-inflamatória exacerbada, aumentando o risco de infecção secundária ${ }^{18}$.

Alguns dos eventos mais importantes associados à resposta inflamatória (SIRS) são a liberação de citocinas, como Interleucinas (IL-1 e IL-6) e fator de necrose tumoral (TNF), e as alterações hormonais. Ocorre resistência à insulina e ao hormônio do crescimento $(\mathrm{GH})$ e redução na secreção de testosterona e do fator de crescimento insulina-like-1 (IGF-1), responsável pela síntese protéica no fígado e musculatura esquelética. Por outro lado, há aumento nas concentrações dos hormônios contra-reguladores, como as catecolaminas, glucagon e cortisol ${ }^{19}$.

$\mathrm{Na}$ fase da resposta compensatória (CARS), há produção de citocinas antiinflamatórias, como IL-4, IL-10, IL-13 e TGF $\beta$ (transforming growth factor), que induzem, principalmente, à imunoparalisia ${ }^{18}$. Provavelmente, tais alterações sejam exacerbadas pelo câncer, que também é responsável por modificações metabólicas importantes ${ }^{12,13}$.

Toda essa cascata de eventos metabólicos favorece o catabolismo das proteínas, carboidratos e lipídios endógenos, com a finalidade de prover substratos intermediários essenciais e energia, necessários para gerar combustível para os processos envolvidos na resposta à agressão. Esses fatores, portanto, são responsáveis pelo hipercatabolismo e hipermetabolismo observados em pacientes graves com câncer.

\section{Alterações no metabolismo energético durante o estresse}

Pacientes gravemente doentes são acometidos por alterações no metabolismo dos carboidratos, lipídios e proteínas (Figura 1). Essas alterações promovem o aumento das necessidades energéticas e catabolismo protéico, e contribuem para alterações no sistema imune e trato gastrointestinal. A resposta de fase aguda ao estresse é, provavelmente, designada para produzir energia e substratos para a síntese protéica e reparação celular nos tecidos viscerais - gastrintestinal, fígado, células imunes etc. - e nos locais acometidos pela doença ou em processo de cicatrização ${ }^{19}$. 
Outros Tecidos

Cérebro, pulmão, coração, eritrócitos, etc. não insulino-dependente

$\uparrow$ Captação de glicose

$\uparrow$ Oxidação de glicose
Músculo esquelético
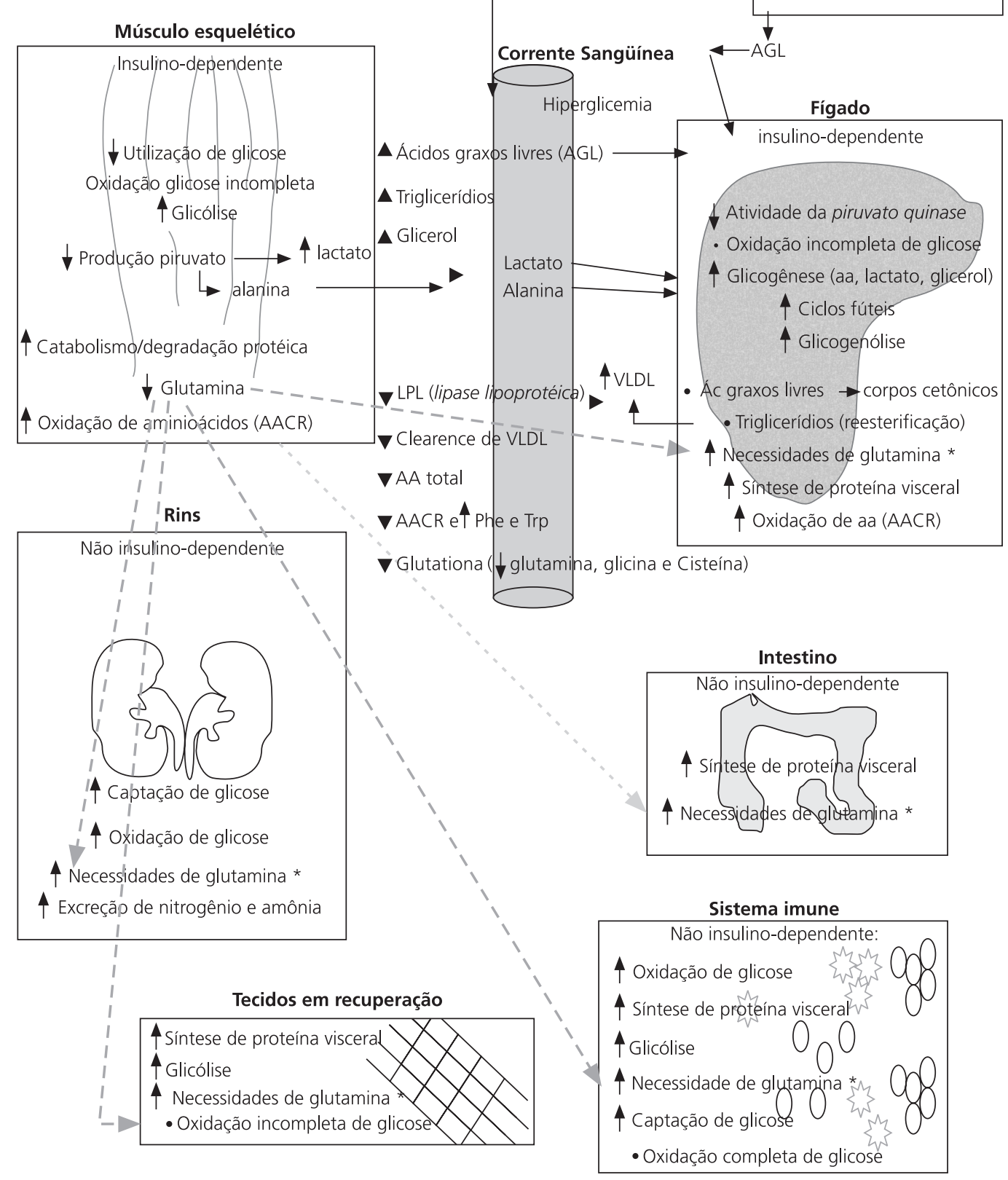

Figura 1. Alterações no metabolismo energético no doente crítico durante a fase de estresse. Modificado de Biolo19 . *Precursor da gliconeogênese, substrato para proliferação/reparação celular, síntese de nucleotídeos, produção de glutationa, excreção da amônia. $A A C R=$ aminoácidos de cadeia ramificada; Phe= fenilalanina; $\operatorname{Trp}=$ triptofano. 
Nessa situação ocorre aumento no turnover protéico total, pois tanto o catabolismo como o anabolismo estão exacerbados. A degradação protéica no músculo esquelético oferece suporte para o aumento na síntese de proteínas de fase aguda no fígado e fornecimento de aminoácidos para os tecidos e órgãos vitais. Esse fenômeno é essencial para oferecer condições ao hospedeiro de suportar o processo do estresse metabólico e caracteriza o balanço nitrogenado negativo, observado freqüentemente nessa situação. Não obstante, durante o estresse prolongado e intenso, cujo catabolismo é de difícil controle, a depleção grave nos estoques orgânicos pode prejudicar a recuperação da doença e influenciar negativamente a morbimortalidade dos pacientes.

Assim, a terapia nutricional não é capaz de conter totalmente a depleção da proteína muscular, mas a oferta nutricional adequada, associada aos cuidados metabólicos, pode minimizar o processo ${ }^{17,19}$.

\section{Complicações decorrentes da oferta inadequada de energia}

A administração inadequada de nutrientes nessa situação tem justificado alguns cuidados específicos, principalmente para evitar o excesso de energia pela via endovenosa. Com esse intuito, alguns autores preconizam a monitorização sistemática do gasto energético em pacientes críticos por meio da utilização de métodos calorimétricos, que apresentam maior precisão para essas medidas ${ }^{20,21}$.

Atualmente, o uso da calorimetria indireta em pacientes críticos tem se tornado rotina nos grandes centros de tratamento do mundo. Entretanto, a maioria das unidades de tratamento para pacientes críticos não dispõe desse recurso, o que torna o indivíduo mais exposto aos riscos da hipo e da hiperalimentação ${ }^{21}$. Nos últimos anos, o excesso de oferta de energia e nutrientes tem sido mais freqüentemente observado do que a oferta insuficiente em pacientes criticamente doentes ${ }^{22}$. Essas complicações elevam o custo do tratamento de pacientes graves em aproximadamente $\$ 5,19$ por paciente/dia, justificando a aquisição do equipamento também para reduzir o desperdício. Esses recursos têm demonstrado importância na redução das complicações da estimativa nutricional inadequada, principalmente pela via parenteral ${ }^{23}$. Apesar de extensivamente preconizada, a calorimetria é um equipamento de custo relativamente elevado, o que dificulta sua aquisição pela maioria das instituições.

O conceito de hiperalimentação ou overfeeding relaciona-se à provisão de energia e/ou substratos energéticos em excesso, ou seja, além das necessidades para manter a homeostase metabólica ${ }^{17}$. Durante o estado de estresse agudo, a natureza da enfermidade pode ser responsável por uma variedade de respostas metabólicas. Além disso, pode ocorrer variabilidade nas respostas interindividuais para o mesmo tipo de doença e nas respostas intra-individuais em função da progressão ou regressão do quadro clínico, implicando alterações do gasto energético. Os efeitos prejudiciais da hiperalimentação resultam, principalmente, em complicações metabólicas, aumento do gasto energético, comprometimento respiratório, disfunção hepática, supressão imunológica e aumento da morbimortalidade ${ }^{24}$.

A mortalidade tem sido associada à sobrecarga de energia, principalmente de carboidratos. O efeito da sobrecarga de energia por meio da administração de glicose foi avaliado em indivíduos no pós-operatório com base nos valores de quociente respiratório $(\mathrm{QR})$, considerando-se QR $>0,95$ como grupo normocalórico (150\% do gasto energético) versus $\mathrm{QR}<0,95$ como grupo hipocalórico ( $100 \%$ do gasto energético). O número de pacientes que desenvolveu sepse e a taxa de mortalidade foram significativamente maiores no grupo hiperalimentado em relação ao grupo que recebeu quantidade de energia igual à taxa metabólica medida pela calorimetria (100\% versus $40 \%$ e $28 \%$ versus $10 \%$, respectivamente $)^{25}$. Esses dados confirmam a importância da avaliação adequada do gasto energético. 


\section{TERA PIA NUTRICIONAL}

\section{Aspectos gerais}

A maioria dos pacientes com câncer está sob grande risco de desenvolver agravo da condição clínica em qualquer momento durante o tratamento antineoplásico, especialmente pela maior suscetibilidade do sistema imune e debilidade orgânica associadas às toxicidades das drogas e outras complicações terapêuticas. $\mathrm{Na}$ vigência desse quadro, há a necessidade de um tratamento intensivo de suporte, que inclui, entre outros, o suporte nutricional.

A terapia nutricional tem como objetivos: oferecer condições favoráveis para o estabelecimento do plano terapêutico; oferecer energia, fluidos e nutrientes em quantidades adequadas para manter as funções vitais e a homeostase; recuperar a atividade do sistema imune; reduzir os riscos da hiperalimentação; garantir as ofertas protéica e energética adequadas para minimizar o catabolismo protéico e a perda nitrogenada.

Quanto ao método de terapia nutricional, a nutrição enteral, por meio de suplementos orais, sondas nasogástricas, nasojejunais ou de gastrostomia/jejunostomia, e a NP, por via periférica ou central, são os métodos comumente utilizados. A escolha do método mais adequado dependerá da situação que envolve o paciente. Portanto, o melhor método é aquele que, considerando as diferentes circunstâncias, proporciona menor risco e maior eficiência para a obtenção do melhor custo-benefício, além da complacência do paciente e seus familiares e experiência da equipe multidisciplinar.

Algumas condições foram estabelecidas para auxiliar na indicação da terapia nutricional: perda de peso superior a $5 \%$ em relação ao peso anterior ao diagnóstico; relação de peso e estatura inferior a $90 \%$ ou ao percentil 10; comprometimento de albumina ou, preferivelmente, pré-albumina; redução das reservas adiposas: prega cutânea triciptal inferior ao percentil 5; redução de dois percentis no peso ou estatura; ingestão alimentar menor que 70\% das necessidades por cinco dias, independentemente do déficit antropométrico ou outras condições; distúrbio ou toxicidade gastrointestinal por cinco dias, independentemente do déficit antropométrico ou outras condições. Na Figura 2 está representado algoritmo para decisão da via de administração ${ }^{26-29}$.

\section{Nutrição enteral}

A utilização de terapia nutricional por via enteral em crianças com câncer, por meio de sondas nasoentéricas ou gastrostomias, apresentou um aumento importante na última década nos países mais desenvolvidos. Essa mudança na conduta reduziu o uso da nutrição parenteral total (NPT) e os riscos associados a ela, com resultados animadores quanto à recuperação e/ou manutenção do estado nutricional. Atualmente, a indicação precoce de nutrição enteral é um dos principais objetivos da terapia nutricional em crianças e adolescentes com câncer. O Comitê de Cuidados de Suporte do Grupo de Estudos de Crianças com Câncer (CCSG) recomenda que o TGI seja a via de escolha para o suporte nutricional dessas, deixando a NPT reservada para os casos em que a nutrição enteral, mesmo associada à NP periférica, seja inadequada ou contra-indicada ${ }^{29-32}$.

As indicações mais freqüentes para uso de sonda em crianças com câncer em situação grave estão descritas no Quadro 1, juntamente com situações mais comuns que contra-indicam a via enteral nesses pacientes. Essas, normalmente, são temporárias, mas necessitam ser identificadas rapidamente para prevenir o agravo nutricional, que pode ocorrer por ocasião de jejuns prolongados $26,33,34$.

A maioria dos pacientes graves deve ser alimentada precocemente, dentro de 24 a 48 horas. No entanto, esse processo exige uma adequada ressuscitação e restauração da perfusão mesentérica. Por isso, alguns indicadores descritos a seguir são utilizados para nortear tal decisão ${ }^{34}$. estabilização hemodinâmica; fluxo esplâncnico e perfusão da mucosa intestinal adequados; redução da necessidade de fluidos e sangue; correção dos distúrbios ácido-básicos. 


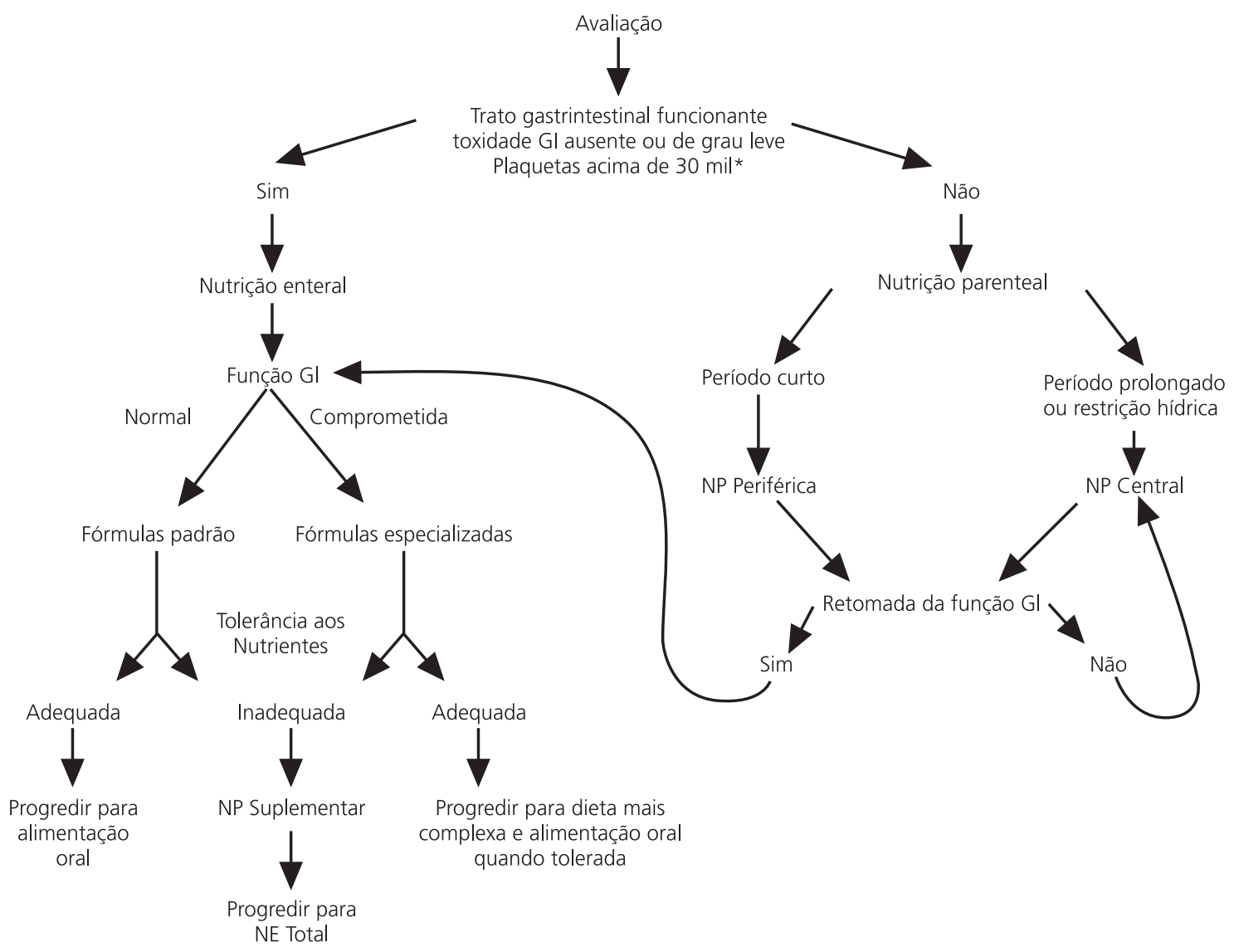

Figura 2. Algoritmo para decisão da terapia nutricional especializada (TNE).

Modificado de Sacks \& Meek ${ }^{26}$, ASPEN ${ }^{28}$ e de Bowman et al. ${ }^{29} .{ }^{*}$ Toxicidade gastrintestinal (GI)= náuseas e vômitos, mucosite, constipação e diarréia grau leve=1 e 2 (não contra-indica a passagem de sonda); grau grave= 3 e 4 (contra-indica a passagem de sonda). Contagem de plaquetas inferior a $30 \mathrm{mil}$ células $/ \mathrm{mm}^{3}$ tem maior risco de sangramento e perfuração $\mathrm{Gl}$, requerendo infusão para o procedimento de passagem de sonda.

Quadro 1. Indicações e contra-indicações da nutrição enteral por sonda em crianças graves com câncer.

\begin{tabular}{|c|c|}
\hline Indicações & Contra-indicações \\
\hline $\begin{array}{l}\text { 1. Pacientes com desnutrição leve ou perda de peso entre } 5 \% \text { e } \\
10 \% \text {, apresentando ingestão alimentar com suplementação oral } \\
\text { inferior a } 70 \text { - } 80 \% \text { das necessidades de energia por } 3 \text { a } 5 \text { dias. } \\
\text { 2. Redução da superfície intestinal absortiva por má absorção devido } \\
\text { à quimioterapia, radioterapia, ressecção intestinal ou diarréia. } \\
\text { 3. Desnutrição grave ou perda de peso recente superior a 10\%. } \\
\text { 4. Estados de hipercatabolismo, como sepse e cirurgia. } \\
\text { 5. Comprometimento neurológico e/ou alto risco de broncoaspiração: } \\
\text { - disfagia/incapacidade de sucção ou deglutição; } \\
\text { - pós-operatório neurocirúrgico complicado; } \\
\text { - ventilação pulmonar mecânica; } \\
\text { - insuficiência respiratória. }\end{array}$ & $\begin{array}{l}\text { 1. Íleo paralítico } \\
\text { 2. Obstrução intestinal } \\
\text { 3. Hemorragias gastrintestinais } \\
\text { 4. Inflamação intestinal } \\
\text { 5. Peritonite } \\
\text { 6. Choque séptico ou hipovolêmico } \\
\text { 7. Má perfusão ou isquemia intestinal } \\
\text { 8. Plaquetopenia grave que não resolve com infusão } \\
\text { de plaquetas } \\
\text { 9. Toxicidade gastrintestinal de grau } 3 \text { ou } 4 \text { (mucosite, } \\
\text { vômitos incoercíveis e diarréia intratável que pioram } \\
\text { com a oferta de dieta). }\end{array}$ \\
\hline
\end{tabular}


Definir critérios para avaliar a eficácia da terapia nutricional enteral implantada é importante para identificar os pacientes que realmente poderão se beneficiar da NP ${ }^{34}$. Assim, a indicação de NP deve ser considerada quando a nutrição enteral por sonda não alcançar $50 \%$ das necessidades após 72 horas ou $70 \%$ das necessidades após sete dias. Essa também poderá ser associada à nutrição por sonda, caso o paciente apresente intolerância com a progressão da dieta enteral. Crianças com câncer são mais suscetíveis ao desenvolvimento de intolerâncias e toxicidades gastrintestinais devido ao tratamento oncológico. O risco desses efeitos é potencializado quando há agravo da condição clínica. Algumas condutas $^{26,28,35,36}$ que podem auxiliar no sucesso da nutrição enteral no paciente crítico com câncer estão descritas no Quadro 2.

As principais complicações do uso de sondas em crianças com câncer podem ser de ordem mecânica, gastrintestinal e infecciosa ${ }^{37-40}$. As mecânicas incluem obstrução da sonda por administração de medicamentos ou lavagem inadequada após infusão da dieta, sinusite, esofagite e necrose de asa do nariz com o uso prolongado, deslocamento da sonda por vômitos, broncoaspiração e perfuração gastrintestinal com risco de sangramento intenso. Quanto às compli-

Quadro 2. Cuidados com a alimentação enteral em crianças gravemente doentes.

1 Realizar confirmação radiográfica do posicionamento da sonda.

2 Administrar antieméticos durante a terapia antineoplásica que apresenta risco de provocar náuseas e vômitos.

3 Alguns pacientes podem se beneficiar do uso de agentes pró-cinéticos para aumentar tolerância à alimentação gástrica e como profilaxia do estresse.

4 Efetuar avaliação do volume de resíduo gástrico $\otimes$ associado à vigilância de sinais clínicos de intolerância em pacientes recebendo alimentação por SNG.

5 Optar por sonda pós-pilórica (abaixo do ligamento de Treitz), quando identificar pacientes com intolerância à alimentação gástricaభ ou com risco de aspiração"•. Caso seja necessário, encaminhar para endoscopia ou fluoroscopia.

6 Indicar gastrostomia/jejunostomia quando a terapia nutricional necessitar de tempo mais prolongado com a sonda.

7 Utilizar sondas de calibre mais fino e material flexível (poliuretano ou silicone), para reduzir o risco de lesão do TGI e intolerância Gl.

8 Implantar terapia nutricional precoce, efetuando o procedimento de inserção da sonda ou colocação da gastrostomia antes do aparecimento de lesões em mucosa Gl.

9 Realizar o procedimento de colocação de sonda fora dos períodos de trombocitopenia grave.

10 Realizar infusão de plaquetas antes do procedimento de colocação de sonda, quando a contagem for inferior a 30 mil células $/ \mathrm{mm}^{3}$, para reduzir o risco de lesão e sangramento da mucosa gastrintestinal.

11 Utilizar bomba de infusão para administrar a dieta e preferir gotejamento contínuo.

12 Optar por dietas industrializadas, moduladas se necessário, com baixa osmolaridade e isentas de lactose.

13 Pacientes com intolerância a dietas poliméricas devem fazer uso de dietas semi-elementares.

14 Monitorizar constantemente procedimentos de higiene, preparo e administração, eventuais da dieta, complicações gastrintestinais e controles de posicionamento e fixação da sonda.

15 Efetuar irrigação da sonda com água após cada infusão de dieta intermitente, após a administração de medicamentos e a cada 4 horas durante a alimentação contínua.

Fontes: Sacks \& Meek ${ }^{26} ;$ Aspen ${ }^{28} ;$ McClave et al. ${ }^{35}$, Peltz $^{51}$.

SNG= sonda nasogástrica; $\mathrm{TGI}=$ trato gastrintestinal; $\mathrm{Gl}=$ gastrintestinal.

$\otimes$ Necessitam de monitorização do volume de resíduo gástrico os pacientes com rebaixamento do nível de consciência e redução da velocidade do esvaziamento gástrico, considerando-se que um volume persistentemente alto seja de risco para aspiração.

భ Pacientes em quimioterapia, compressão gástrica pelo tumor e ascite são suscetíveis para intolerância à alimentação gástrica.

-• Fatores de risco para aspiração: redução do nível de consciência (Glasgow Coma Scale <9), posição de supinação, sonda nasogástrica, sonda mal posicionada, intubação endotraqueal ou ventilação mecânica, sedação, vômitos, volume de resíduo gástrico persistentemente alto, administração da dieta em bolo ou intermitente, sondas de grande calibre, doenças ou condições que promovem retardo no esvaziamento gástrico (doença neurológica, cirurgia torácica ou abdominal, diabetes melitos, hiperglicemia, distúrbio eletrolítico). 
cações gastrintestinais, destacam-se os vômitos associados à quimioterapia, retardo do esvaziamento gástrico ou aumento na velocidade de infusão da dieta e diarréia e distensão abdominal por intolerância à dieta, quimioterapia ou radioterapia ou por redução da perfusão da mucosa intestinal. As complicações de ordem infecciosa relacionam-se à contaminação microbiana acidental durante o processo de manipulação da dieta ou durante sua administração, podendo causar diarréia, desidratação e infecção. No caso do uso de estomias, as principais complicações são inflamação local, celulite e infecção.

\section{Nutrição parenteral}

A nutrição parenteral total (NPT) em pacientes com câncer tem motivado grande discussão na literatura. A necessidade de nutrir o paciente com câncer, que freqüentemente apresenta algum grau de desnutrição associada às dificuldades da alimentação pela via enteral devido às toxicidades gastrointestinais, leva ao maior uso de NPT. Entretanto, a imunossupressão, decorrente do tratamento antineoplásico, põe esses indivíduos sob maior risco de desenvolver infecções associadas à NPT. Assim, definir situações em que a via endovenosa trará mais benefícios do que prejuízos é fundamental para o planejamento terapêutico desses pacientes. A seguir serão discutidos alguns aspectos teóricos que sustentam as bases das diretrizes atuais.

O uso de terapia nutricional em pacientes com câncer visa melhorar a resposta ao tratamento, as chances de cura e a sobrevida, sem, entretanto, aumentar os riscos. Muitos estudos têm sido publicados nas últimas décadas com o intuito de definir o papel da NPT nesses pacientes. Com a finalidade de interpretar adequadamente tais resultados, alguns comitês e sociedades desenvolveram análises sistemáticas desses dados.

A análise do American College of Physicians sobre doze estudos randomizados controlados com pacientes em quimioterapia concluiu não haver benefício da NPT quanto à resposta tumoral, demonstrando redução na sobrevida global e na sobrevida em três meses. Além disso, no mesmo estudo observou-se aumento de quatro vezes no número de infecções no grupo que recebeu NPT. Entretanto, a heterogeneidade quanto aos diagnósticos e quanto às condições de administração da NPT enfraquece os resultados ${ }^{41}$.

Outra análise, que incluiu 28 estudos prospectivos randomizados de pacientes em quimioterapia e radioterapia, não demonstrou benefício quanto à sobrevida, tolerância ou toxicidade do tratamento e resposta do tumor à terapia antineoplásica. Ainda, no mesmo estudo, observou-se aumento do risco de infecção ${ }^{42}$. Em crianças submetidas à radioterapia pélvica ou abdominal, a NPT também não demonstrou diferença na sobrevida, apesar da recuperação nutricional43.

Entretanto, pacientes submetidos à quimioterapia intensiva para transplante de medula óssea (TMO) demonstram se beneficiar com a $\mathrm{NPT}^{43,44}$. Num estudo prospectivo com 137 pacientes randomizados para receber ou não NPT, demonstrou-se diferença significante com aumento da sobrevida global, sobrevida livre de doença e do tempo para recaída com o uso da terapia ${ }^{45}$. Quando os mesmos pacientes foram estratificados quanto ao tipo de TMO, o grupo que recebeu transplante alogênico e NPT profilática demonstrou aumento da sobrevida em doze $\operatorname{anos}^{40}$.

Esses estudos, apesar de algumas críticas metodológicas, como a heterogeneidade da amostra, quanto ao diagnóstico de câncer, estado nutricional e tipo de terapia nutricional, serviram de base para as diretrizes atuais, que desencorajam a administração rotineira de NPT em pacientes com câncer quando outra via pode ser utilizada para efetuar o suporte nutricional.

Pacientes TMO apresentam benefícios mais definidos com a NPT, provavelmente pelos altos índices e gravidade da toxicidade gastrointestinal, que impede, numa grande porcentagem dos casos, a utilização da nutrição enteral por sondas ${ }^{46}$. 
Por outro lado, em pacientes com câncer do trato gastrointestinal, o uso de NPT no pré-operatório tem demonstrado reduzir as taxas de complicações e mortalidade. Sua oferta para pacientes com câncer gastrointestinal por sete a dez dias antes da cirurgia demonstra reduzir as complicações pós-operatórias em aproximadamente $10 \%$ dos $\operatorname{casos}^{43,47}$.

Entretanto, num estudo prospectivo, The Veterans Affairs Total Parenteral Cooperative Study Group, foram acompanhados 192 pacientes de onze hospitais (66,0\% com vários tipos de câncer), randomizados para receber NPT pré-operatória sete a dez dias antes até 72 horas após a cirurgia ou não $(n=203)$. Todos os pacientes foram acompanhados por 90 dias após a cirurgia. Os resultados demonstraram aumento das complicações infecciosas no grupo com NPT (14,0\% versus $6,4 \% ; p<0,01)$, não havendo diferença, porém, nas taxas de complicações não-infecciosas graves e mortalidade. Quando os pacientes foram estratificados quanto ao estado nutricional, houve uma correlação entre o grau de desnutrição e a eficácia da NPT, demonstrando que pacientes com desnutrição mais grave tiveram menos complicações não-infecciosas com o uso da terapia $(22,6 \% \text { versus } 42,0 \% ; p<0,03)^{48}$.

Em outro estudo, pacientes com câncer de trato gastrointestinal que receberam dez dias de NPT pré-operatória apresentaram redução de 30\% nas taxas de complicações e redução da mortalidade quando gravemente desnutridos ${ }^{49}$. Recentemente, Heyland et al..$^{50}$ realizaram uma meta-análise com 27 ensaios clínicos randomizados com NPT no período pré-operatório de várias enfermidades, incluindo câncer, e concluíram que as taxas de complicações gerais tenderam a ser mais baixas em pacientes desnutridos $(p<0,066)$.

A desnutrição grave é caracterizada por um organismo exaurido de energia e deficiente de reserva nutricional, o que compromete a maioria dos sistemas orgânicos do hospedeiro e justifica os resultados favoráveis da NPT em indivíduos desnutridos. Os principais sistemas acometidos pela desnutrição são o gastrintestinal, o hematopoiético e o imunológico, os mais sensíveis, também, ao tratamento oncológico. Da mesma forma, alterações na função pulmonar, cardíaca, hepática, pancreática, renal e linforreticular têm sido documentadas na desnutrição grave ${ }^{7}$. Assim, algumas adaptações fisiológicas podem ajudar na preservação da vida de indivíduos desnutridos, porém quando essa situação está presente na criança com câncer, os sistemas são afetados também pela terapia antineoplásica. Por isso, o metabolismo de alguns órgãos pode sofrer comprometimento importante, como déficit na habilidade hepática de metabolizar agentes quimioterápicos, do trato Gl em absorver drogas e nutrientes e das células imunes na defesa contra infecções microbianas ${ }^{27}$.

As conclusões dos estudos sugerem que a NPT administrada como rotina no tratamento de pacientes com câncer não apresenta efeito benéfico, mas que, pacientes com desnutrição grave, principalmente no pré-operatório, e, por fim, pacientes TMO podem se beneficiar da $\mathrm{NPT}^{43,48-51}$. Considerando que na maioria dos ensaios clínicos a desnutrição não foi analisada, há a necessidade de mais estudos para identificar os efeitos da NPT nos diferentes graus de comprometimento nutricional.

Como existem poucos estudos em crianças com câncer usando NPT, até o momento não é possível estabelecer conclusões precisas para o grupo. Assim, os resultados dos estudos discutidos anteriormente fundamentam os princípios da terapia nutricional em crianças e adolescentes com câncer. As principais considerações para o uso de NPT são: o uso rotineiro em pacientes em quimioterapia deve ser desencorajado; a maioria dos estudos não incluiu pacientes desnutridos graves, portanto o efeito da NPT nesse grupo ainda deve ser mais bem estudado; outros ensaios clínicos com NPT devem ser encorajados para avaliar efeitos distintos dos obtidos nos estudos anteriores ou em grupos distintos, como crianças e pacientes desnutridos e, por fim, pacientes TMO podem se beneficiar, principalmente quando a NE está contra-indicada. 
Assim, algumas diretrizes para o uso de NPT em pacientes com câncer durante o tratamento antineoplásico e pré-cirúrgico estão listadas a seguir ${ }^{47,52,53}$ : desnutridos graves; pacientes com toxicidade gastrointestinal de graus 3 e 4; qualquer toxicidade orgânica que impossibilite a alimentação oral ou enteral adequada por mais de uma semana; quando a nutrição enteral, mesmo associada à nutrição parenteral periférica, for insuficiente ou contra-indicada; pacientes com caquexia, que não toleram o tratamento antineoplásico sem um período curto de suporte nutricional, auxiliado pela NPT; pacientes eutróficos ou moderadamente desnutridos em quimioterapia, radioterapia ou cirurgia, somente quando houver comprometimento da ingestão alimentar e impossibilidade de NE. A NPT pré-operatória está indicada somente para pacientes com desnutrição grave, por um período de sete a dez dias no mínimo; a NPT não confere benefício em pacientes com câncer avançado, quando documentada falta de resposta ao tratamento antineoplásico.

As complicações mais freqüentemente encontradas com a NP em pacientes com câncer são de origem infecciosa, metabólica ou mecânica. Entre as complicações infecciosas, a contaminação do cateter, principalmente em via central, a manipulação das soluções de NP, e a técnica de assepsia inadequada são as principais causas. Também podem ocorrer alterações de glicemia, hiperosmolaridade, hipertrigliceridemia, distúrbio hidro-eletrolítico, elevação de enzimas hepáticas e deficiência de micronutrientes, entre as complicações metabólicas mais freqüentes. Finalmente, complicações mecânicas, como pneumotórax decorrente da punção e cateterismo venoso central, bem como trombose venosa associada à osmolaridade e $\mathrm{pH}$ da solução, material e tempo de permanência do cateter e tempo de infusão da solução são igualmente importantes.

\section{Avaliação das necessidades nutricionais e recomendações}

As necessidades de energia e nutrientes para crianças e adolescentes variam em função da faixa etária. Na criança e no adolescente, a energia é direcionada para a manutenção das necessidades metabólicas e para o crescimento e desenvolvimento. As necessidades metabólicas incluem taxa de metabolismo basal (TMB) e perda de calor para o meio ambiente. Em relação ao adulto, as necessidades na infância e adolescência são três a quatro vezes maiores, sendo que uma parcela substancial da energia diária ingerida (30\% a 40\%) é necessária para o crescimento nos primeiros seis meses de vida, declinando para $2 \%$ a $5 \%$ aos dois anos. Isso significa dizer que a TMB é de aproximadamente 50 a $55 \mathrm{kcal} / \mathrm{kg}$ na infância e gradualmente declina para 20 a $25 \mathrm{kcal} / \mathrm{kg}$ por dia durante a adolescência.

Embora crianças com doenças graves em cuidados intensivos possam apresentar aumento do gasto energético devido ao estresse metabólico, elas não utilizam energia para o crescimento e para atividade física. Assim, o cálculo do gasto energético deve considerar a taxa metabólica basal, respeitando as limitações metabólicas do estresse agudo e contemplando um acréscimo, somente para compensar a agressão. A necessidade de energia, porém, tende a aumentar durante o período de convalescença ${ }^{17}$.

Apesar das vantagens da medida de calorimetria, o equipamento é especializado e de custo elevado, o que não permite seu uso rotineiro. Assim, algumas equações auxiliam no cálculo dessas estimativas, sendo preconizada para crianças com câncer a da Organização Mundial da Saúde ${ }^{54,55}$.

A oferta adequada de substrato energético, concomitante à adequada oferta protéica, é necessária para assegurar a sua utilização. A relação de energia não protéica para cada grama de nitrogênio deve estar entre 100 e 150:1 para garantir a oferta protéica e evitar a hiperalimentação (Quadro 3) 12,17,26,39,40.

A necessidade de água está relacionada ao gasto de energia, podendo ser determinada por meio do peso corporal. Como as perdas 
insensíveis equivalem a $40 \mathrm{~mL} / 100 \mathrm{kcal}$ e as perdas urinárias a $60 \mathrm{~mL} / 100 \mathrm{kcal}$ em 24 horas, assume-se que $100 \mathrm{~mL} / 100 \mathrm{kcal}$ (ou $1 \mathrm{~mL} / \mathrm{kcal}$ ) são necessários para repor a perda de fluidos em 24 horas (Quadro 3)26,28.

Algumas situações exigem ajustes dessas perdas, quando elas aumentam intensamente, como em processos diarréicos graves, e em condições de febre, quando se estima uma perda insensível de fluido via respiração e pele de $5 \mathrm{~mL} / \mathrm{kg}$ por 24 horas para cada grau de temperatura acima de $38^{\circ} \mathrm{C}$. Perder ou ganhar peso abruptamente quase sempre ocasiona alterações de fluidos, devendo-se efetuar correção ou controle, respectivamente ${ }^{28}$.

As necessidades de eletrólitos estão baseadas nas perdas urinárias de crianças saudáveis (Quadro 3). Da mesma forma que os fluidos, perdas não usuais de eletrólitos, que podem ocorrer devido à diarréia, presença de estomias, terapia diurética e anormalidades renais devem ser consideradas para reposição ${ }^{54}$.

Necessidades específicas de vitaminas e elementos-traço para crianças e adolescentes com câncer não foram estabelecidas. Assim, as recomendações propostas pela ASPEN ${ }^{26}$ são as mais adequadas para a via parenteral, e as Dietary Reference Intakes (DRI), para a via enteral nos cuidados da criança grave ${ }^{28,54}$. Alguns estudos evidenciaram deficiências de nutrientes durante o tratamento oncológico, provavelmente devido ao aumento nas perdas e maior consumo, associado ao estresse oxidativo da radio e quimioterapia. Desse modo, os nutrientes com função antioxidante têm recebido atenção especial. Por isso, no planejamento da terapia nutricional, $\beta$-caroteno, vitaminas A, C e E, zinco, cobre e selênio devem ser ofertados, no mínimo, para alcançar as necessidades. Mais estudos são necessários para estabelecer os benefícios da suplementação de tais nutrientes ${ }^{54}$.

Quadro 3. Necessidades diárias de proteínas, eletrólitos e água.

\begin{tabular}{|c|c|c|c|c|}
\hline \multicolumn{5}{|c|}{ Necessidades protéicas para crianças e adolescentes criticamente doentes } \\
\hline Faixa etária & \multicolumn{4}{|l|}{ Necessidades } \\
\hline Neonatos até 2 anos & \multicolumn{4}{|c|}{2,5 a $3,0 \mathrm{~g} / \mathrm{kg} / \mathrm{dia}$} \\
\hline Crianças (2 a 11 anos) & \multicolumn{4}{|c|}{$2,0 \mathrm{~g} / \mathrm{kg} / \mathrm{dia}$} \\
\hline Adolescentes (acima de 12 anos) & \multicolumn{4}{|c|}{1,5 a $2,0 \mathrm{~g} / \mathrm{kg} / \mathrm{dia}$} \\
\hline \multicolumn{5}{|c|}{$\begin{array}{l}\text { Necessidades diárias de eletrólitos para pacientes } \\
\text { pediátricos ( } \mathrm{mEq} / \mathrm{kg} / \mathrm{dia})\end{array}$} \\
\hline Faixa etária & Pré-termo & Termo & 1 a 10 anos & $>10$ anos \\
\hline Sódio & $2-3$ & $2-5$ & $2-3$ & $2-3$ \\
\hline Potássio & $2-3$ & $1-4$ & $2-5$ & $2-3$ \\
\hline Cloreto & $2-3$ & $1-5$ & $1-2,5$ & $10-20$ \\
\hline Cálcio & $3-4,5$ & $3-4$ & $0,7-1,4$ & $0,7-1,4$ \\
\hline Fósforo & $2,7-4,0$ & $1,5-3,0$ & $0,7-1,4$ & $0,7-1,4$ \\
\hline Magnésio & $0,35-0,6$ & $0,3-0,5$ & $0,3-0,5$ & $10-30$ \\
\hline \multicolumn{5}{|c|}{$\begin{array}{l}\text { Fósforo }(\mathrm{mmol} / \mathrm{kg}) \text { : neonatos }=1-2 ; \text { crianças }=0,5-1 \\
\text { adolescentes }=10-40\end{array}$} \\
\hline \multicolumn{5}{|l|}{ Necessidades diárias de fluidos } \\
\hline$<500 \mathrm{~g}$ & \multicolumn{4}{|l|}{$130-150 \mathrm{~mL} / \mathrm{kg}$} \\
\hline $1500-2000 g$ & \multicolumn{4}{|l|}{$110-130 \mathrm{~mL} / \mathrm{kg}$} \\
\hline Crianças de 3 a $10 \mathrm{~kg}$ & \multicolumn{4}{|c|}{$100 \mathrm{~mL} / \mathrm{kg}$} \\
\hline Crianças de 10 a $20 \mathrm{~kg}$ & \multicolumn{4}{|c|}{$1000 \mathrm{~mL}+50 \mathrm{~mL} / \mathrm{kg}$ para cada $\mathrm{kg}$ acima de $10 \mathrm{~kg}$} \\
\hline Crianças com mais de $20 \mathrm{~kg}$ & \multicolumn{4}{|c|}{$1500 \mathrm{~mL}+20 \mathrm{~mL} / \mathrm{kg}$ para cada $\mathrm{kg}$ acima de $20 \mathrm{~kg}$} \\
\hline
\end{tabular}

Fontes: Weisdorf \& Scharzenberg ${ }^{12}$, Sacks ${ }^{26}$, ASPEN $^{28}$, Garófolo et al. ${ }^{39}$, Weisdorf-Schindele ${ }^{40}$ e Serra ${ }^{53}$.

Equação para o cálculo da relação $\mathrm{kcal} / \mathrm{grama}$ de nitrogênio $=(\mathrm{kcal}$ do lipídeo $+\mathrm{kcal}$ do carboidrato $) \div$ grama total de proteína $\times 0,16 \mathrm{~g}$ (nitrogênio). 


\section{O N C L U S Ã O}

Atualmente, existe pouca experiência no tratamento de crianças com câncer em cuidados intensivos no que se refere à terapia nutricional. Grande parte das informações obtidas vem de estudos com crianças e adultos criticamente doentes com outras enfermidades e de adultos durante o tratamento de câncer ou TMO.

Apesar disso, o progresso tecnológico e científico e o desenvolvimento de novas drogas e técnicas diagnosticadas estão possibilitando maior sobrevida para esses pacientes. Muitos deles recebem tratamento agressivo (quimioterapia, radioterapia, cirurgia e TMO) durante vários anos, estando, portanto, mais expostos aos seus riscos. Infecções e sepse, toxicidades ou falências orgânicas, desnutrição e complicações metabólicas são os principais distúrbios. Assim, o desenvolvimento da ciência da terapia de suporte, como o tratamento das infecções, a terapia intensiva e a terapia metabólico-nutricional, faz-se cada vez mais necessário para que se possam ampliar as possibilidades de cura de crianças e adolescentes com câncer.

Finalmente, o incentivo aos estudos específicos com pacientes gravemente doentes com câncer pode ser de particular importância no desenvolvimento dessa ciência e na aquisição de experiência, especialmente em âmbito nacional.

\section{REFERÊ NCIAS}

1. Petrilli AS, Caran EM, Luisi FA, Barros KVT, Silva NS. Diagnóstico precoce do câncer infantil. Temas de Pediatria-Nestlé. 1992; 50:1-13.

2. Braga PE, Latorre MRDO, Curado MP. Câncer na infância: análise comparativa da incidência, mortalidade e sobrevida em Goiânia (Brasil) e outros países. Cad Saúde Pública. 2002; 18(1):33-44.

3. Lee MLM. Leucemias agudas na infância. Pediatr Mod. 1999; 35:616-21.

4. Viana MB, et al. Malnutrition as a prognostic factor in lymphoblastic leukaemia: a multivariate analysis. Arch Dis Child. 1994; 71:304-10.

5. Aranguré JMM, et al. Malnutrition in childhood lymphoblastic leukemia: a predictor of early mortality during the induction-to-remission phase of the treatment. Arch Med Res. 1999; 30:150-3.

6. Pedrosa F, Bonilla M, Liu A, Smith K, Davis D, Ribeiro RC, et al. Effect of malnutrition at time of diagnosis on the suvival of children treated for cancer in El Salvador and northern Brazil. J Pediatr Hematol Oncol. 2000; 22(6):502-5.

7. Rickard KA, Grosfeld JL, Coates TD, Weetman R. Advances in nutrition care of children with neoplastic diseases: a review of treatment, research, and application. Continuing Educ. 1986; 86(12):106-76.

8. Garófolo A. Estado nutricional de crianças e adolescentes com câncer [dissertação]. São Paulo: Universidade Federal de São Paulo; 2000.

9. Garófolo A, Lopez FA, Petrilli AS. Acompanhamento do estado nutricional de pacientes com osteossarcoma. Acta Oncol Bras. 2002; 22(1): 233-7.

10. Strasser SI, McDonald GB. Gastrointestinal and hepatic complications. In: Thomas ED, Blume KG, Forman SJ. Hematopoietic cell transplantation. 2nd ed. Philadelphia: Blackwell; 1999. p.627-48

11. Papadoupoulou A. Nutritional considerations in children undergoing bone marrow transplantation. Eur J Clin Nutr. 1998; 52(12): 863-71.

12. Weisdorf SS. Nutritional support of hematopoietic stem cell recipients. In: Thomas ED, Blume KG, Forman SJ. Hematopoietic cell transplantation 2nd ed. Philadelphia: Blackwell; 1999. p.723-32.

13. Muscaritoli M, Grieco G, Capria S, lori AP, Fanelli FR. Nutritional and metaboic support in patients undergoing bone marrow transplantation. Am J Clin Nutr. 2002; 75(2):183-90.

14. den Broeder E, Lippens RJ, van't Hof MA, Tolboom $J$ J, Sengers RC, van den Berg AM, et al. Nasogasric tube feeding with cancer: the effect of two different formulas on weight, body composition, and serum protein concentrations. JPEN. 2000; 24(6):351-60

15. Papadopoulou A, MacDonald A, Williams MD, Darbyshike PJ, Booth IW. Enteral nutrition after bone marrow transplantation. Arch Dis Child. 1997; 77(2):131-6.

16. Raynard B, Nitemberg G, Gory-Delabaere G, Bourhis JH, Bachmann PRJ, Bensadoum RJ, et al. Standards, options et recommandations pour la nutrition artificielle au cours et au décours de la greffe de cellules souches hématopiétiques (CSH). Bull Cancer. 2002; 89(4):381-98.

17. Chwals WJ. Overfeeding the critically ill child: factor fantasy? New Horizon. 1994; 2(2):147-55. 
18. Grimminger F, Seeger W, Mayer K. Use of n-3 fatty acid-containing lipid emulsion in the Intensive Care Unit environment: the clinician's view. Clin Nutr. 2002; 21(S2):23-9.

19. Biolo G, Grimble G, Preiser JC, Leverne $X$, Jolliet $P$, Planas $M$, et al. Position paper of the ESICM Working Group on Nutrition and Metabolism. Metabolic basis of nutrition in intensive care unit patients: ten critical questions. Intensive Care Med. 2002; 28(11):1512-20.

20. Porter C, Cohen NH. Indirect calorimetry in critically ill patients: role of the clinical dietitian in interpreting results. J Am Diet Assoc. 1996; 96(1):49-57.

21. McClave AS, McClain CJ, Snider HL. Should indirect calorimetry be used as part of nutritional assessment? J Clin Gastroenterol. 2001; 33(1):14-9.

22. Frankenfield DC, Wiles CE, Bagley S, Siegel JH. Relationships between resting and total energy expenditure in injured and septic patients. Crit Care Med. 1994; 22(11):1796-804.

23. McClave AS, Lowen CC, Kleber MJ, Nicholson JF, Jimmerson SC, McConnell JW, et al. Are patients fed appropriately according to their caloric requirements? JPEN. 1998; 22(6):375-81.

24. Klein CJ, Stanek GS, Wiles CE. Overfeeding macronutrients to critically ill adults: metabolic complications. J Am Diet Assoc. 1998; 98(7): 795-806.

25. Vo NM, Wayscater M, Acuff RV, Lefemine AA. Effects of postoperative carbohydrate overfeeding. Am Surg. 1987; 53(11):632-5.

26. Sacks N, Meek R. Nutritional support. In: Ablin AR. Current therapy and guidelines from the children's cancer group: "supportive care of children with cancer". Baltimore (MD): Jonhs Hopkins University Press; 1997. p.193-209.

27. Andrassy RJ, Chwals WJ. Nutritional support of the pediatric oncology patient. Nutrition. 1998; 14(1):124-9.

28. ASPEN Board of Directors and The Clinical Guidelines Task Force. Guidelines for the use of parenteral and enteral nutrition in adult and pediatric patients. JPEN. 2002; 26(1 Suppl): 15A-1385A, 26(2):144.

29. Bowman LC, Williams R, Sanders M, RingwaldSmith K, Baker D, Gajjar A. Algorithm for nutritional support: experience of the metabolic and infusion support service of St. Jude Children's Research Hospital. Int J Cancer. 1998; 11:76-80.

30. Heubi JE. Whenever possible, use the gut! J Pediatr Hematol Oncol. 1999; 21(2):8-9.
31. Pietch JB, Ford C. Whitlock JA. Nasogastric tube feeding in children with high-risk cancer: a pilot study. J Pediatr Hematol Oncol. 1999;21(2): 111-4.

32. Baron MA, et al. Efficacy and safety of radiologically placed gastrostomy tubes in pediatric hematology/oncology patients. Med Pediatr Oncol. 2000; 34:177-82.

33. Kirby DF. Decisions for enteral access in the intensive care unit. Nutrition. 2001; 17(9):776-9.

34. Spain DA. When is the seriously ill patient ready to be fed? JPEN. 2002; 26(6):S62-8.

35. McClave AS, DeMco MT, Delegge MH, DiSaria JÁ, Heybaud DK, Maloney JP, et al. North American Summit on Aspiration in the Critically III Patient: consensus statement. JPEN. 2002; 26(6 Suppl):S80-5.

36. Metheny NA. Risk factors for aspiration. JPEN. 2002; 26(6):S26-33.

37. George DL, Falk OS, Umberto Meduri G, Sepper KVJr, Wunderink RG, Steere EL, et al. Nosocomial sinusitis in patients in the medical intensive care unit: a prospective epidemiologica study. Clin Infect Dis. 1998; 27(3):463-70.

38. Barbosa MCM, Barbosa AP. Nutrição enteral em pediatria. In: Ferro HC, Azevedo JRA, Loss SH. Nutrição enteral e parenteral em UTI. Rio de Janeiro: Atheneu; 2001. p.349-81

39. Garófolo A, Ancona Lopez F, Petrilli AS. Terapia nutricional em oncologia pediátrica. Pediatr Mod. 2001; 37(9):413-27.

40. Weisdorf-Schindele S, Schwarzenberg SJ. Nutritional support of hematopoietic cell recipients. In: Blume KG, Forman SJ, Appelbaum F. Thoma's hematopoietic cell transplantation. 3rd ed. Philadelphia: Blackwell; 2004. p.883-93

41. McGeer AJ, Detsky AS, O'Rourke K. Parenteral nutrition in patients receiving cancer chemotherapy. Ann Intern Med. 1989;110(9): 734-6.

42. Klein S, Simes J, Blackburn GL. Total parenteral nutrition in cancer clinical trials. Cancer. 1986; 58(6):1378-81.

43. Harrison LE, Brennan MF. The role of total parenteral nutrition in the patient with cancer. Curr Prob Surg. 1995; 32(10):833-924.

44. Cohen J, Lefor AT. Nutrition support and cancer. Nutrition. 2001; 17(7/8):698-9.

45. Weisdorf AS, Lysne J, Wind D, Haake RJ, Sharp HL, Goldman A, et al. Positive Effect of prophylactic total parenteral nutrition on long-term outcome of bone marrow transplantation. Transplantation. 1987; 43(6):833-8. 
46. Sefcick A, Anderton D, Byrne JL, Teahon K, Russell $\mathrm{NH}$. Naso-jejunal feeding in allogeneic bone marrow transplant recipients: results of a pilot study. Bone Marrow Transplantation. 2001; 28:1135-9.

47. Rombeau JL, McClane SJ. Perioperative care of the colorectal patient. Dis Colon Rectum. 1999; 42(7):845-56.

48. Buzby GP, et al. The Veterans Affairs Total Parenteral Nutrition Cooperative Study Group. Perioperative total parenteral nutrition in surgical patients. $\mathrm{N}$ Engl J Med. 1991; 325(8):525-32.

49. Chung A. Perioperative nutrition support. Nutrition. 2002; 18(2):207-8.

50. Heyland DK, Montalvo M, MacDonald S, Keefe L, Su XY, Drover JW. Total parenteral nutrition in the surgical patient: a meta-analysis. Can J Surg. 2001; 44(2):102-11.

51. Peltz G. Nutrition support in cancer patients: a brief review and suggestion for standard indications criteria. Nutrition J. [serial on the Internet]. 2002 [cited 2003 Jan 5]; 1:[about 1p.] Available from: http:IIwww.nutritionj.com/content/2/1/18

52. ASPEN. Board of Directors and The Clinical Guidelines. Safe practices for parenteral nutrition formulations. National Advisory Group Standards and Practice Guidelines for Parenteral Nutrition. Special Report. JPEN. 1998; 22(2):1-41.

53. Serra AS. Parenteral nutrition and the surgical patients. Barcelona: Danone Chair MonographsInstitut Danone; 1999. 193p.

54. Garófolo A. Avaliação nutricional e requerimentos. In: Petrilli AS, Carvalho BW, Lee JH. Cuidados intensivos no paciente oncológico pediátrico. São Paulo: Atheneu; 2003. p.213-30.

55. Children's Oncology Group Symposium. Cancer Control-Nutrition Sub-Committee. Algorithm for nutrition intervention and categories of nutritional status in the pediatric oncology patient-references and resources. Washington (DC); COG; 2004.

Recebido para publicação em 18 de setembro de 2003 e aceito em 24 de agosto de 2004. 